\title{
Modelling for Control of a Steckel Hot Rolling Mill
}

\author{
E. SCHOLTZ, I. K. CRAIG ${ }^{1)}$ and P. C. PISTORIUS ${ }^{21}$
}

Department of Electrical and Electronic Engineering, University of Pretoria, Pretoria 0002 South Africa and Department of Electrical Engineering and Computer Science, Massachusetts Institute of Technology, Cambridge, MA 02139, USA. E-mail:escholtz@mit.edu. 1) Department of Electrical and Electronic Engineering, University of Pretoria, Pretoria 0002 South Africa. E-mail: icraig@postino.up.ac.za. $\quad$ 2) Department of Materials Science and Metallurgical Engineering, University of Pretoria, Pretoria 0002 South Africa. E-mail: pistori@postino.up.ac.za.

(Received on February 28, 2000; accepted in final form on May 29, 2000)

In this work the derivation of a nonlinear plant simulator of a Steckel Hot Rolling Mill process is shown. The simulator reflects the thickness crown and tension behaviour of the strip, while the temperature, shape and flatness behaviour of the strip fall outside the scope of this work. Roll gap, stand, tension and hydraulic actuator models are incorporated in order to yield the simulator. The nonlinear simulator is used to identify a linear model for control system design. The linear model was identified at a certain operating point associated with a particular pass of a multiple pass rolling schedule. Step tests were applied to the manipulated variables of the simulator, and using system identification (SID) techniques a linear time invariant (LTI) multivariable input output (MIMO) transfer function model was identified. Lastly an initial control problem is formulated.

KEY WORDS: Steckel rolling mill; hot rolling; mill simulator; nonlinear dynamic modelling; system identification; LTI MIMO model; control problem formulation; centerline gauge; tension; strip crown.

\section{Introduction}

Over capacity of steel production has increased manufacturing competition, causing tighter quality margins to become the norm for the market. A need for automation and improved control arises to satisfy the emphasis placed on improved dimensional accuracy, surface finish, flatness, and physical properties, ${ }^{1)}$ without a decline in throughput. Rolling mill automation projects focus on material tracking, automatic slab and coil storage control and reheating furnace control, automatic control, and data logging. ${ }^{1)}$ More emphasis is placed on the automatic control of: mill sequencing; mill-setup and correction; pass schedule calculation/optimization; thickness control; ${ }^{2-4}$ crown and shape control, ${ }^{5)}$ finishing temperature and the strip's mechanical properties. ${ }^{6)}$ Initial investigations into the application of advanced control methods in the rolling mill environment, to help with achieving these requirements have been conducted $\left(H_{\infty},{ }^{7)}\right.$ Generalized Predictive Control (GPC), ${ }^{8)}$ Observer based controller ${ }^{2)}$ ).

This work is the result of the initial phase of a research project investigating the application of Model Predictive Control (MPC) to the Steckel hot rolling mill. It focuses on modelling for thickness profile and tension control. ${ }^{9)}$ The derivation of nonlinear models from the literature and first principles is done in order to construct a simulator. This approach is necessitated due to the unavailability of non-proprietary models and/or suitable data.

The regulation of the mill outputs at threading speed have been the main research area for quite some time, while control of head and tail ends of the strip are now being investigated. $^{7,10)}$ The benefit of control on the head and tail end of the strip is that the amount of off-specification product can be reduced. ${ }^{10)}$ The Steckel hot rolling mill process is a batch process with multiple process startups and stops associated with one strip making the investigation into process start-ups imperative.

\section{Process Description}

Rolling is the process of shaping metal by passing it between rolls revolving at the same peripheral speed and in opposite directions. The Steckel mill (Fig. 1) is a single stand, reversing mill consisting of two work and back up rolls. Steckel Mills are scarce and are mainly found in developing countries due to their relatively low investment costs. ${ }^{1)}$ This mill employs multiple passes to achieve the desired final thickness of the strip. The reversing of the strip during the multiple passes results in a temperature decline, which increases the rolling forces. The temperature rundown is compensated for by winding the strip on coiler drums situated in furnaces regulated at a temperature of $970^{\circ} \mathrm{C}$.

The aim of thickness control is to regulate the exit thickness $\left(h_{2}([\mathrm{~m}])\right)$ from the roll gap within customer specified quality accuracies. Improved control can decrease the thickness standard deviation and move the mean of $h_{2}$ closer to the lower specified thickness bound, yielding a material saving for the producer. ${ }^{10)}$ The control of the tension in the strip whilst rolling is a regulator problem. ${ }^{11)}$ If the deviation goes uncontrolled and the tension in the strip is higher than the material's yield stress, necking occur and the strip can ultimately tear. As discussed in this paper, tension also affects material flow in the roll gap.

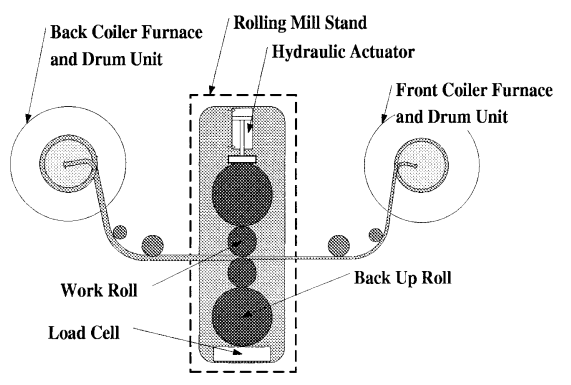

Fig. 1. Steckel hot rolling mill. 


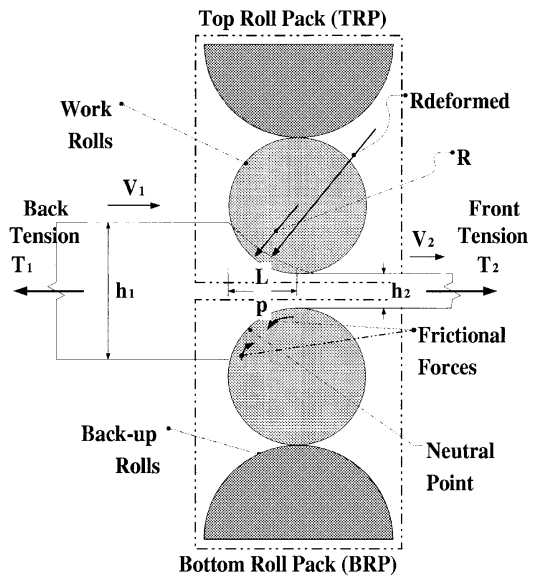

Fig. 2. Roll gap.

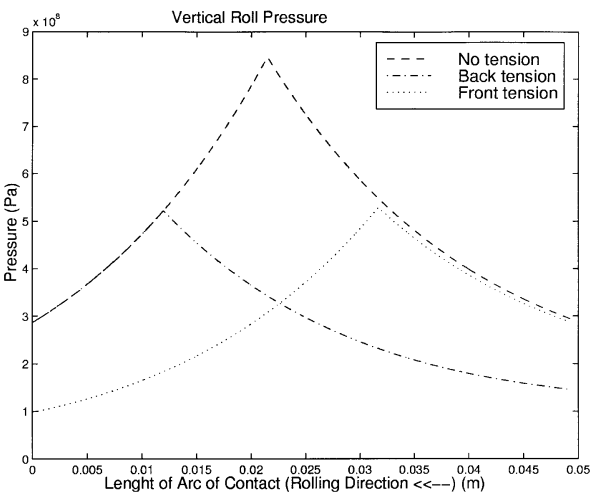

Fig. 3. Vertical roll pressure distribution along the roll gap distance as a function of the applied strip tensions. This figure was constructed from simulation data.

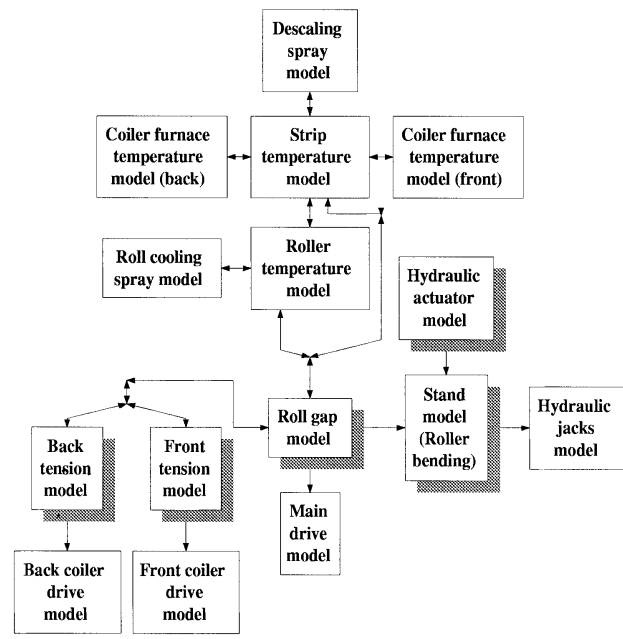

Fig. 4. Models identified in order to constitute a comprehensive nonlinear mill simulator.

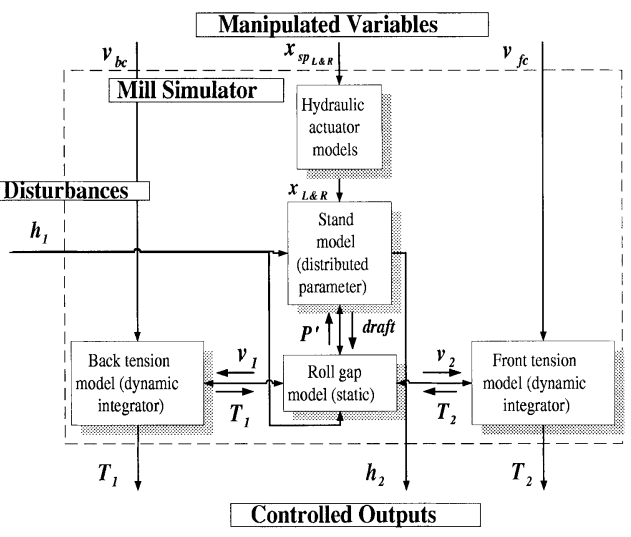

Fig. 5. Plant/simulator $\mathrm{I} / \mathrm{O}$ relationship.

measured plant temperatures, and $T_{1}$ and $T_{2}$.

Back and Front Tension Models (TM): The evolution of $T_{1}$ and $T_{2}$ is accounted for. The inputs are $v_{1}$ and $v_{2}$, obtained from the RGM and the manipulated speed variables of the back and front coiler motors $\left(v_{b c}\right.$ and $\left.v_{f c}\right)$. The outputs are the controlled variables $T_{1}$ and $T_{2}$, which are fed back to the RGM, making $T_{1}$ and $T_{2}$ also disturbances.

Stand Model (SM): The mill stretch and the roll bending are accounted for. The spatial modelling of the rollers provides the simulator with a draft deviation across the width of the sheet and thus the strip thickness profile. The inputs are $P^{\prime}$ across the width of the strip, and the cylinder chamber pressures of the hydraulic actuators $\left(P_{L 1}, P_{L 2}, P_{R 1}, P_{R 2}\right.$ $([\mathrm{Pa}]))$. The output of this model is a draft deviation*2: $\delta(z, t)=\delta_{\text {setup }}-\left(\delta h_{1}(t)-\left(y_{1}(z, t)-y^{2}(z, t)\right)\right)$.

Hydraulic Actuators Models (HA): These actuators are the primary devices employed to control $h_{2}$. These models are intertwined with the SM to create a nonlinear statespace model, which forms one part of the simulator.

A lack of some of the identified models are compensated for by using practical measured data as input to the mill simulator. The temperatures measured by pyrometers outside the roll gap are linearly interpolated to yield an estimate of the roll gap temperature. The main mill drive speed

*1 $L_{p}$ reflects a transport delay across the roll gap.

*2 With $-w / 2 \leq z \leq w / 2, \delta h_{1}(t)$ is the measured centerline entrance thickness of the strip, $\delta h_{2}(z, t)$ is the exit thickness of the strip calculated over the width of the strip (w). $y_{1}$ and $y_{2}([\mathrm{~m}])$ are the vertical displacement of the TRP and BRP. It is assumed that the strip is very ductile and fills the whole roll gap. The simulator can thus only account for strip with an entering positive (convex) crown that is contact with the rolls at all time. 
is not a manipulated variable and is obtained from measured data. The measured data of pass $3\left(h_{1}=13 \mathrm{~mm}\right.$ and $h_{2}=9 \mathrm{~mm}$ ) of a 5 pass industrial Steckel mill schedule rolling grade 304 stainless steel strip, was tailored to complement the models of the simulator. The operating point of the simulator was chosen on the mill speed up ramp at a speed of $3.5 \mathrm{~m} / \mathrm{s}$ with $\theta \approx 970^{\circ} \mathrm{C}$. Logged data of pass 3 incorporates effects of control actions taken in previous passes, thus complicating the extraction of open loop data from closed loop data.

In what follows $i \in[1,2], s \in[L, R], \varrho \in[1,2], \chi \in[T, B]$ and $l \in[1,2]$ are indices $* 3$. The appearance of double signs in an equation should be interpreted as two equations, where the top sign corresponds to the first element(s) of the appropriate indice-set(s) and the bottom sign to the second element(s). See also Nomenclature.

\subsection{Roll Gap Model}

\subsubsection{Model}

Available roll gap models are of the analytical and numerical finite element type. The analytical models are static and used to predict the rolling force and torque, examples being the models of Orowan ${ }^{12}$ and Sims. The Finite Element models either predict the rolling force and torque or the material behaviour. ${ }^{6}$ In this work Orowan's model is adapted as a roll gap model. ${ }^{12)}$

The rolling load is a function of the material yield stress $(k(\bar{\varepsilon}, \dot{\varepsilon}, \theta)([\mathrm{Pa}]))$, rolling geometry $\left(R, R^{\prime}, L_{\mathrm{p}}, \phi\right)$, friction coefficient $(\mu)^{* 4}$ and the applied tensions to the strip $\left(T_{1}\right.$ and $T_{2}$ ). The material yield stress is a function of the type of material being rolled, its temperature, the draft taken and the rolling speed $v_{\text {roll }}([\mathrm{m} / \mathrm{s}])$.

The Zener-Holoman flow stress $(\bar{\sigma})$ model takes the work hardening of the strip and the strain rate sensitivity of the material into account. $\bar{\sigma}$ is then,

$$
\bar{\sigma}=\frac{\sqrt{3} k(\varepsilon, \dot{\varepsilon}, \theta)}{2}=A_{Z H} \bar{\varepsilon}^{n} \overline{\dot{\varepsilon}}^{m} \exp \left(\frac{Q_{Z H}}{R_{\text {ideal }} \theta}\right) .
$$

Horizontal and vertical force balances are equated on an infinitesimal roll gap element, yielding a differential equation from the horizontal force balance and a relationship between the forces from the vertical force balance. The Huber-Mises yield criterion (see Eq. (2)) accounts for the plastic deformation in the roll gap, ${ }^{12)}$ yielding a relation between the vertical roll pressure $p$ ([Pa]), horizontal force $f$ ([N]) and $k(\bar{\varepsilon}, \overline{\dot{\varepsilon}}, \theta)$. Combining the mentioned relationships in the differential equation yields Orowan's standard differential equation, shown in Eq. (3). The thickness of the strip $(h(\phi))$ can be calculated at any point along the arc of contact for a 2D $x-y$ plane (see Eq. (4)), and the spatial origin is defined at the exit of the roll gap $\phi \triangleq 0$ ) increasing against the rolling direction. The thickness of the sheet $(h(\phi))$ at any point in the roll gap is expressed in Eq. (4). Substituting Eq. (4) into Eq. (3), a modified version of Orowan's differential equation, which is used in the simulator, is obtained. ${ }^{* 5}$

$$
\begin{aligned}
& p(\phi)-\frac{f(\phi)}{h(\phi)}=k(\varepsilon, \dot{\varepsilon}, \theta) . \\
& \frac{d f}{d \phi}=2 R^{\prime}\left[\frac{f}{h}+k(\varepsilon, \dot{\varepsilon}, \theta)\right]\left[\frac{\sin \phi \pm \mu \cos \phi}{1 \mp \mu \tan \phi}\right] . \\
& h=h_{2}+2 R^{\prime}(1-\cos \phi) \text {. } \\
& \frac{d p}{d \phi}=\frac{2 R^{\prime}}{h}\left[p\left(\frac{\sin \phi \pm \mu \cos \phi}{1 \mp \mu \tan \phi}\right)-(p-k) \sin \phi\right] \ldots
\end{aligned}
$$

\subsubsection{Simulation Methodology}

The roll pressure $p$ causes the work rolls to flatten along the arc of contact. This flattened arc can be modelled as a larger circular radius with a shifted centre. ${ }^{12)} P^{\prime * 6}$, is a function of $R^{\prime}$, which depends on the amount of roll gap deformation caused by $P^{\prime}$. This implicit relationship necessitates an iterative solution process in order to solve for $P^{\prime}$ and $R^{\prime}$. Hitchcock's formula provides the necessary relationship: ${ }^{12,13)}$

$$
R^{\prime}=R\left[1+\frac{16\left(1-v^{2}\right) P^{\prime}}{\pi E \delta}\right]
$$

The two independent ODEs in Eq. (5) are each solved numerically in a Gauss-Seidel iterative scheme (where $R^{\prime}$ is updated) from either side of the roll gap until they equate at the neutral point. The following boundary conditions are used,

$$
p(\phi)+\sigma_{i}=k(\bar{\varepsilon}, \overline{\dot{\varepsilon}}, \theta)
$$

with $\left.\phi\right|_{i=1}=\phi_{m}$ and $\left.\phi\right|_{i=2}=0$.

$\delta(z, t)$ varies across the width of the sheet due to the bending of the rollers and thus the RGM is simulated for multiple $x-y$ slices across the width of the strip.

\subsection{Tension Model}

\subsubsection{Model}

A base tension is required in the strip in order to reap the benefit of a reduced rolling load and can be calculated using, ${ }^{9,15)}$

$$
T_{i}=47 w h_{i}^{0.27}, \quad \forall i \in[1,2] .
$$

When the roll gap distance is changed, the draft of the strip varies, changing $v_{1}$ and $v_{2}$. The strip pieces were modelled as lumped spring elements and these speed changes cause the tensions to vary according to, ${ }^{11)}$

$$
T_{i}=\frac{E_{s s}\left(h_{i} w_{\text {strip }}\right)}{L_{c f \leftrightarrow r g}} \int_{0}^{t}\left( \pm v_{i}(\tau) \mp v_{c}(\tau)\right) d \tau
$$

where $\left.E_{s s}\right|_{\theta=970^{\circ} \mathrm{C}}=106 \times 10^{9} \mathrm{~Pa}$ is Young's modulus for grade 304 stainless steel, $\left.v_{c}\right|_{i=1}=v_{b c},\left.v_{c}\right|_{i=2}=v_{f c}([\mathrm{~m} / \mathrm{s}])$, and $L_{c f \leftrightarrow r g}=6.4 \mathrm{~m}$ is the length from the coiler furnaces to the roll gap.

\footnotetext{
*3 $i$ reflects which side of the roll gap is under investigation, e.g. Front or Back. $s$ reflects which side of the roll stand is under investigation, e.g. Left or Right. $\varrho$ and $\chi$ reflect which roll pack is under investigation, e.g. Top or Bottom. $\imath$ reflects which chamber of the hydraulic cylinder is under investigation, e.g. Rod or Piston side.

${ }^{* 4}$ For hot rolling the friction coefficients at the work rolls and strip interfaces are between $0.25 \leq \mu \leq 0.45 .{ }^{6)}$ In the simulator the friction coefficient is calculated as a linear function of $\theta,{ }^{14)} \mu(\theta)=4.86 \times 10^{-4} \theta-0.0714$.

$* 5$ For the sake of brevity the notation that the variables are functions of $\phi$ are not shown.

${ }^{* 6} P^{\prime}=R^{\prime} \int_{0}^{\phi_{m}} p(\phi) d \phi$.
} 
It is assumed that the base tension is established before the mill motor is accelerated towards the threading speed of a particular pass. The large gains of the pure capacitive processes specify the bandwidth of the processes. ${ }^{16)}$ The main control aim is to regulate the tension in the sheet around the setup tension value, and it is not desirable that the strip should neck or vibrate transversely due to over-relaxation of tension*7. At threading speed the tangential speed of the strip is controlled by manipulating $v_{b c}$ and $v_{f c}$ such that the tangential speeds remain the same as $v_{1}$ and $v_{2}$. The radii of the front/back coils are increasing/ decreasing with time, and the angular velocities of the coiler motors are controlled according to, $\omega_{\text {coilerdrive }}=$ $v_{\text {tangential }} / R_{\text {coil \& drum }}$.

\subsubsection{Simulation Methodology}

The simulation frequency of the model lowers when the thickness decreases (see Eq. (10)). This shows that tension control is of increasing importance with decreasing thickness, when the bandwidths of the tension process and the hydraulic actuator systems become comparable. The simulation frequency was made 2 times larger than the system bandwidth. ${ }^{16)}$

$$
\omega_{T M}=\frac{E_{s s} w h_{i}}{L_{c f \leftrightarrow r g}}=\frac{106 \times 10^{9} w h_{i}}{6.4},
$$

The input is the draft in the middle of the sheet, $\delta$ $\left(z=0, t=t_{0}\right)$. The tension loop is solved at a higher frequency than the rest of the models, with $\Delta t_{\mathrm{TM}}<\Delta t_{\text {Main }}$.

The tension module is solved only for the centerline gauge and has two loops:

- An outer time loop that is simulated for time $t=$ $t_{0}+n \Delta t_{T M}, \forall t_{0} \leq t \leq t_{0}+\Delta t_{\text {Main }} * 8$.

- An inner loop accounting for an iterative solution of $T_{1}$ and $T_{2}$ as a function of $v_{1}$ and $v_{2},\left.P^{\prime}\right|_{z=0},\left.R^{\prime}\right|_{z=0}$. The execution of this loop is stopped after the GaussSeidel filtered tension value has stabilized to within a $0.1 \%$ error of the normalized tension differences between two iterative steps.

\subsection{Stand Model}

The stand model (Fig. 6) is modelled as a distributed parameter system with four continuous beam masses that interact with discrete lumped elements. The stand model is described in an asymmetrical fashion with regards to the horizontal plane through the roll gap ${ }^{9}$ as well as around a vertical plane through the mill centerline. ${ }^{2,9)}$ The vibration effect due to changing draft and the variation of the friction coefficient with temperature are taken into account through the RGM.

The assymetrical behaviour around the mill centerline can be attributed to the chattering of the mill, ${ }^{17)}$ as well as to the difference in the mill spring on both sides of the mill, ${ }^{7,9)}$ In this work asymmetrical behaviour is accounted for by modelling different mill stretch curves for either side of the mill frame. The effect of mill drive spindles chattering is not taken into account in this work.

Different methods have been developed to predict the strip thickness profile and shape. ${ }^{5)}$ The modelling approach used in this work is the elastic foundation method (efm) al-

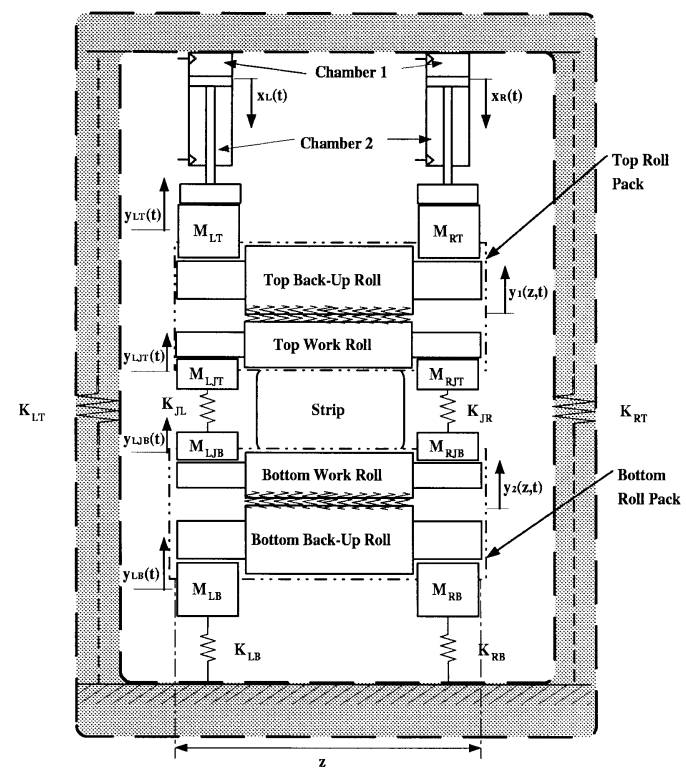

Fig. 6. Cross sectional view of the mill.

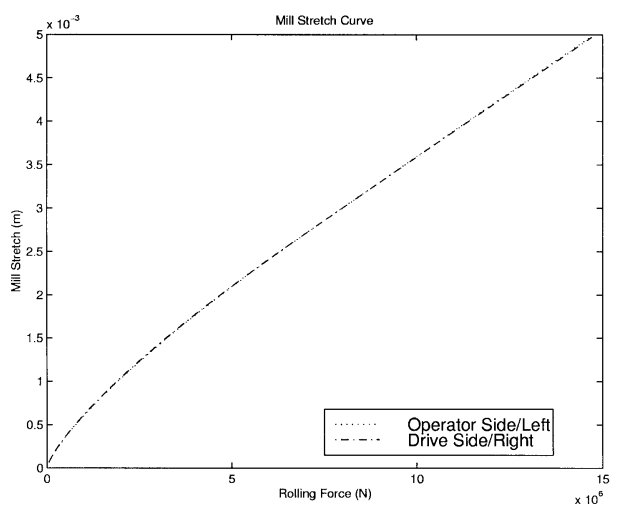

Fig. 7. Constructed mill stretch curves of the practical mill under consideration.

though in Ref. 5) it is mentioned that efm has a limited capability compared to FEM methods. In this work a dynamic model is derived using the efm-modelling approach which performed well in the work of Pederson ${ }^{2}$ who designed an observer based controller for a practical plate mill using an efm approach.

The four beam model is simplified to a two beam model in accordance with Ref. 2) and suggestions made by Dobrucki et al. ${ }^{17)}$ The TRP-Top Roll Pack and BRPBottom Roll Pack are obtained when the top/bottom work and back-up rolls are grouped together. The mill stretch on both sides of the mill is predominantly linear (see Fig. 7).

The PDE model of the two roll pack is $* 9, * 10, * 11$.

$$
\rho A_{\varrho} \frac{\partial^{2} y_{\varrho}}{\partial t^{2}}+E I_{\varrho} \frac{\partial^{4} y_{\varrho}}{\partial x^{4}}= \pm P^{\prime} \mp \beta\left(\frac{\partial y_{1}}{\partial t}-\frac{\partial y_{2}}{\partial t}\right) \text {. }
$$

The boundary values of Eq. (11) are:

\footnotetext{
*7 In this instance tension can be seen as negative and the effect of applied tension to the roll gap model was forced to be zero.

$* 8$ is a simulation counter.

*9 The dependency notation of the variables, $A_{i}, y_{i}, I_{i}, P^{\prime}$, on $z$ and $t$ are omitted for the sake of brevity.

*10 The weight of the rolls were not taken into consideration since the ratio $W / F_{\max }$ is less than $5 \%$ of the maximum rolling force ( $\left.40 \mathrm{MN}\right)$.

*11 Elastic recovery of the strip and the functioning of hydraulic jacks are not modelled as a first approximation in this work.
} 


$$
\begin{gathered}
\left.\frac{\partial y_{\varrho}}{\partial x}\right|_{z=\frac{ \pm l_{\varrho}}{2}}=0 \\
\left.E I_{1} \frac{\partial^{3} y_{1}}{\partial x^{3}}\right|_{z=\frac{\mp l_{1}}{2}}= \pm M_{s T} \frac{\partial^{2} y_{s T}}{\partial t^{2}} \pm F_{H A s} \cdots \\
\left.E I_{2} \frac{\partial^{3} y_{2}}{\partial x^{3}}\right|_{z=\frac{\mp l_{2}}{2}}= \pm M_{s B} \frac{\partial^{2} y_{s B}}{\partial t^{2}} \pm K_{s B} y_{s B} .
\end{gathered}
$$

The Assumed Modes Method is a separation of variables analytical approximate method ${ }^{18)}$ used to solve Eq. (11). The vertical displacement vector $\mathbf{y}(z, t)(\approx \Phi(z) \mathbf{q}(t))$ is approximated by a finite dimensional vector inner product of $\Phi(z)$ (natural modes of vibration) and $\mathbf{q}(t)$ (normal time coordinates). ${ }^{18)}$ Assumed modes $\Psi(z)$ are chosen that adhere to Eq. (12). For the Assumed Modes method the number of modes $(2 \alpha)$ is chosen two times larger than the $(\alpha)$-number predominant spatial modes $* 12$. When the number of desired assumed modes increase with one, the number of states of the stand model increases with 2 for each roller. The assumed modes are,

$$
\begin{aligned}
& \psi_{\varrho 1}(z)=4.25\left(\frac{2 z}{l_{\varrho}}\right)^{3}-1.38\left(\frac{2 z}{l_{\varrho}}\right)-0.5, \\
& \psi_{\varrho_{2}}(z)=0.15\left(\frac{2 z}{l_{\varrho}}\right)^{4}-0.3\left(\frac{2 z}{l_{\varrho}}\right)^{2}+1.0,
\end{aligned}
$$

where $\Psi_{\varrho}=\left[\begin{array}{ll}\psi \varrho_{1} & \psi \varrho_{2}\end{array}\right], \forall \varrho \in[1,2]$, and for the two roll pack, $\Psi=\left[\begin{array}{ll}\psi_{1} & \psi_{2}\end{array}\right]$.

Substituting $\hat{\mathbf{y}}(z, t) \approx \psi(z) \mathbf{q}(t)$ into Eq. (11) and employing Euler integration*13 over the width of the stand the following matrix partial differential equation is obtained: ${ }^{18,19)}$

$$
\begin{gathered}
\underbrace{\Psi_{\varrho}^{T} \Delta_{z} \rho A_{\varrho} \Psi_{\varrho}}_{\mathbf{M}_{\varrho \varrho}} \ddot{\mathbf{q}}_{\varrho}+(\underbrace{\left.\Psi_{\varrho}^{(2)}\right)^{T} \Delta_{z} E I_{\varrho} \Psi_{\varrho}^{(2)}}_{\mathbf{K}_{\varrho \varrho}} \mathbf{q}_{\varrho} \\
+\underbrace{\Psi_{\varrho}^{T} \Delta_{z} \beta \Psi_{\varrho}}_{\mathbf{C}_{\varrho \varrho}} \dot{\mathbf{q}}_{\varrho}=\underbrace{\Psi_{\varrho}^{T} \Delta_{z} \beta \Psi_{\varrho^{c}}}_{\mathbf{C}_{\varrho \varrho} \mathbf{C}} \dot{\mathbf{q}}_{\varrho^{c}} \\
\pm \Psi_{\varrho}^{T} \Delta_{z} P^{\prime} ; \varrho \in[1,2], \varrho^{c} \in[2,1] \ldots
\end{gathered}
$$

The displacements at the end of the rolls and the displacement of the discrete elements are the same, thus providing the connecting relationship between the discrete and the continuous systems. The matrices for the discrete elements are constructed as,

$$
\Gamma_{\mathrm{s}}=\psi_{\varrho}(z)^{T} \gamma_{s} \psi_{\varrho}(z), \quad \in \mathfrak{R}^{2 \alpha \times 2 \alpha},
$$

with $\Gamma_{s} \in\left[\mathbf{M}_{d s i}, \mathbf{M}_{d J s i}, \mathbf{K}_{d s 2}\right]$, correspondingly $\gamma_{s} \in\left[M_{s \chi}\right.$, $\left.M_{J s \chi}, K_{s B}\right]$, and $\left.z\right|_{s=L}=-l_{\varrho} / 2,\left.z\right|_{s=R}=l_{\varrho} / 2$.

When the above matrices are summed the assumed modes mass and stiffness matrices $\left(\in \mathfrak{R}^{4 p \times 4 p}\right)$, are obtained as,

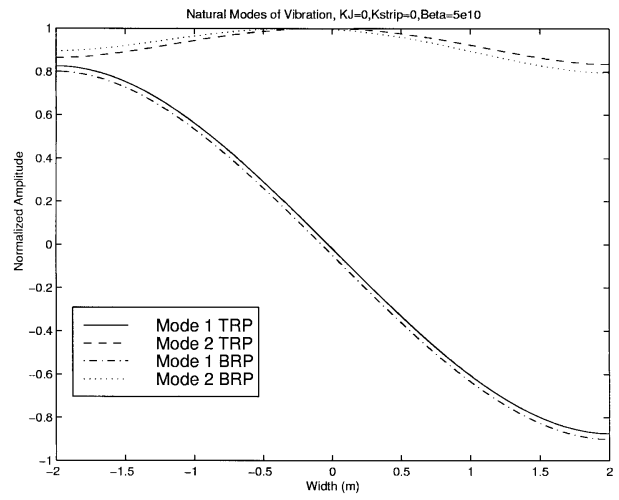

Fig. 8. Natural modes of vibration.

$$
\begin{array}{r}
\hat{\mathbf{M}}=\operatorname{diag}\left(\mathbf{M}_{11}+\mathbf{M}_{d L 1}+\mathbf{M}_{d R 1}+\mathbf{M}_{d J L 1}+\mathbf{M}_{d J R 1}, \mathbf{M}_{22}+\mathbf{M}_{d L 2}\right. \\
\left.+\mathbf{M}_{d R 2}+\mathbf{M}_{d J L 2}+\mathbf{M}_{d J R 2}\right), \ldots \ldots \ldots \ldots \ldots \ldots \ldots \ldots \ldots \ldots . . . \ldots \ldots \ldots \ldots \ldots \ldots \ldots \ldots \ldots \\
\hat{\mathbf{K}}+\operatorname{diag}\left(\mathbf{K}_{11}, \mathbf{K}_{22}+\mathbf{K}_{d L 2}+\mathbf{K}_{d R 2}\right) . \ldots \ldots \ldots \ldots \ldots . . .
\end{array}
$$

The modal matrix $\mathbf{U}$ is obtained by solving a generalized eigenvalue problem,

$$
\left(\hat{\mathbf{K}}-\omega^{2} \hat{\mathbf{M}}\right) \mathbf{U}=0,
$$

where $\mathbf{U} \in \mathfrak{R}^{4 \alpha \times 4 \alpha}$. The spatial solution, $\boldsymbol{\Phi}(z)$, is then obtained by using the relation $(\boldsymbol{\Phi}(z) \in \Psi(z) \mathbf{U})$. The calculated natural modes of vibration are shown in Fig. 8.

Using $\mathbf{U}$ the decoupled mass, stiffness and damping matrices are constructed as, $\mathbf{M}=\mathbf{U}^{T} \hat{\mathbf{M}} \mathbf{U}, \mathbf{K}=\mathbf{U}^{T} \hat{\mathbf{K}} \mathbf{U}, \mathbf{C}=$ $\mathbf{U}^{T} \hat{\mathbf{C}} \mathbf{U}$ and $\mathbf{q}=\left[\begin{array}{llll}q_{1} & q_{1} & q_{2} & q_{2}\end{array}\right]^{T} \in \mathfrak{R}^{2(2 \alpha) \times 1} * 14$.

The decoupled normal coordinate system forms a linear ODE system that is used in the simulator:

$$
\left[\begin{array}{c}
\dot{\mathbf{q}} \\
\ddot{\mathbf{q}}
\end{array}\right]=\left[\begin{array}{cc}
\mathbf{0} & \mathbf{I} \\
-\mathbf{M}^{-1} \mathbf{K} & -\mathbf{M}^{-1} \mathbf{C}
\end{array}\right]\left[\begin{array}{l}
\mathbf{q} \\
\dot{\mathbf{q}}
\end{array}\right]+\left[\begin{array}{c}
\mathbf{0} \\
\mathbf{M}^{-1} \mathbf{U}^{T} \mathbf{B}
\end{array}\right] \mathbf{u}_{\text {sim }} \cdots
$$

where the input matrix and vector are defined as*15,

$$
\begin{aligned}
& \mathbf{B}=\left[\begin{array}{cc}
\Upsilon \Omega & \Delta_{z} \Psi_{1}(z)^{T} \\
\mathbf{0} & -\Delta_{z} \Psi_{2}(z)^{T}
\end{array}\right] \\
& \mathbf{u}_{\text {sim }}=\left[\begin{array}{lllll}
P_{L 1} & P_{R 1} & P_{L 2} & P_{R 2} & P^{\prime}
\end{array}\right]^{T} .
\end{aligned}
$$

In Ref. 3) the lumped damping for the HAGC system is provided between the strip and the working rolls. The corresponding distributed damping is dependent on the geometry of the deformed roll gap as follows,

$$
\beta_{s}(t)=\frac{\left.R^{\prime} k(\varepsilon, \dot{\varepsilon}, \theta)\right|_{\phi=0}}{v_{\text {roll }}}\left[\frac{\mathrm{N}}{\mathrm{m}^{2} \cdot \mathrm{s}^{-1}}\right],
$$

Guo $^{3)}$ showed that there is a contradiction with the calculated values and practical behaviour. In this work Eq. (23) was used due to a lack of substitute model and a calculated $\beta=1.18 \times 10^{8} \mathrm{~N} \cdot \mathrm{m}^{-2} \cdot \mathrm{s}$ was obtained. For this choice bumpless simulation startup did not result and when beta was increased to $5 \times 10^{10}$ an approximate steady state was main-

\footnotetext{
*12 $\alpha=1$ in Refs. 2), 9) and $2 \alpha=3$ in Ref. 17).

$* 1351$ discretized nodes spaced at $\Delta_{z}=8 \mathrm{~cm}$.

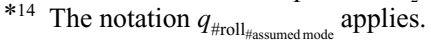

*15 $Y=\left[\Psi_{1}^{T}\left(\frac{-l_{1}}{2}\right) \quad \Psi_{1}^{T}\left(\frac{-l_{1}}{2}\right)\right], \Omega=\left[\begin{array}{cccc}A_{L 1} & 0 & A_{R 1} & 0 \\ 0 & A_{L 2} & 0 & A_{R 2}\end{array}\right]$
} 
tained. The vibration period obtained in Ref. 9) is $2.6 \mathrm{~ms}$, which corresponds to the vibration periods of 3-4 ms given in Ref. 17)

The load cell at the bottom of the mill was modelled as a discrete spring with, $K_{s B}=0.5 F_{\text {limit }} / 0.05 \mathrm{~mm}=4 \times 10^{11} \mathrm{Nm}$.

\subsection{The hydraulic Actuator}

\subsubsection{Model}

The hydraulic actuator system consists of 6 major dynamic components ${ }^{* 16}$ that can be modelled to capture the nonlinear dynamic behaviour of the Hydraulic Actuator Gauge Control (HAGC) system ${ }^{3,20)}$ The hydraulic actuators were modelled from first principles and according to approach suggested by Guo. ${ }^{3)}$ In modern designs the pressure line is kept as short as possible. As a result the transmission line dynamics can be omitted. The sensor dynamics are modelled as unity gains. Similar actuators were modelled on both sides of the mill.

The servovalve ${ }^{3)}$ was modelled as a second order system,

$$
\ddot{x}_{v_{s}}+2 \zeta_{v_{s}} \omega_{v_{s}} \dot{x}_{v_{s}}+\omega_{v_{s}}^{2} x_{v_{s}}=\frac{\omega_{v_{s}}^{2} x_{v_{s} \max }}{I_{o_{s}}} I_{c_{s}} \ldots \ldots
$$

$I_{c}$ is calculated in Eq. (25) by assuming a cascaded PI-controller with $K_{c_{s}}=1.5$ and $\tau_{i_{s}}=1 \times 10^{-2}$. ${ }^{c_{s}}$ )

$$
I_{c_{s}}=K_{c_{s}}\left(\left(x_{s_{s p}}-x_{s}\right)+\frac{1}{\tau_{i_{s}}} \int_{0}^{t}\left(x_{s_{s p}}-x_{s}\right) d \tau\right) \text {. }
$$

A double acting hydraulic actuator was modelled. The direction of positive cylinder flow is defined as flow into both chambers and the leakage flow from chamber 1 (piston side) to chamber 2 (rod side). The following continuity equations are obtained:

$$
Q_{s l}=Q_{s l}(t) \pm \frac{V_{0 s l} \pm A_{s l} x_{s}}{\beta_{o}} \dot{P}_{s l}+A_{s l} \dot{x}_{s}
$$

The servovalve can be charging $\left(x_{v} \geq 0\right)$ or dumping $\left(x_{v}<0\right)$, causing the hydraulic stroke to increase and decrease respectively. The cylinder flows are expressed as,

$$
Q_{s l}(t)=K_{v_{s}} x_{v_{s}}(t) \sqrt{\frac{2}{\rho_{o}} \Delta P_{s l}(t)},
$$

In Table 1 the flow pressure difference in Eq. (27) are given for the two modes of operation. The hydraulic actuators interact with the stand model, and the thrust force of one of these actuators is,

$$
P_{s 1}(t) A_{s 1}-P_{s 2}(t) A_{s 2}=F_{H A s}(t)
$$

The hydraulic actuators are top mounted resulting in a floating actuator base, whose coordinates are determined by the mill stretch.

The relationship between the movement of the upper roll pack, hydraulic stroke and mill stretch is,

$$
x_{s}(t) \approx \frac{\left(P_{s 1}(t) A_{s 1}-P_{s 2}(t) A_{s 2}\right)}{K_{s T}}-y_{s T}(t) .
$$

Taking the derivative of Eq. (29) and substituting this into the second equation generated by Eq. (26) when $l=2$ yields,
Table 1. Pressure difference for the servovalve scenarios.

\begin{tabular}{|c|c|c|}
\hline Case & $\Delta P_{s 1}$ & $\Delta P_{s 2}$ \\
\hline \hline$x_{v_{s}} \geq 0$ & $P_{\text {high }}-P_{s 1}(t)$ & $P_{s 2}(t)-P_{\text {low }}$ \\
\hline$x_{v_{s}}<0$ & $P_{s 1}(t)-P_{\text {low }}$ & $P_{\text {high }}-P_{s 2}(t)$ \\
\hline
\end{tabular}

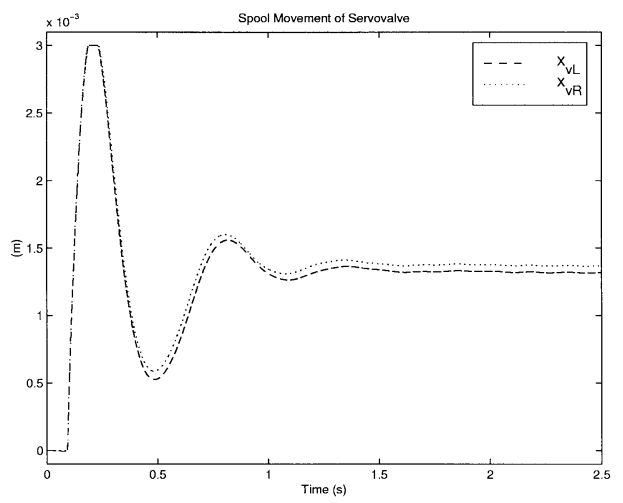

Fig. 9. Servovalve spool stroke, after application of a step $\left.\delta x_{s p_{L \& R}}\right|_{t \geq 92.6 \mathrm{~ms}}=1 \mathrm{~mm}$.

$$
\dot{P}_{s 2}=\frac{-Q_{s 2}+Q_{s l}-A_{s 2} \dot{y}_{s 1}+\frac{\dot{P}_{s 1} A_{s 1} A_{s 2}}{K_{s T}}}{\left(\frac{V_{s 2}\left(x_{s}\right)}{\beta_{o}}+\frac{A_{s 2}^{2}}{K_{s T}}\right)} \ldots \ldots
$$

Defining, $\Delta=V_{s 2}\left(x_{s}\right) / \beta_{o}+A_{s 2}^{2} / K_{s T}$ and $\gamma=A_{s 1} A_{s 2} /\left(K_{s T} \Delta\right)$, and substituting Eq. (30) and the derivative of Eq. (29) into Eq. (26) when $\imath=1$ yields,

$$
\dot{P}_{s 1}=\frac{Q_{s 1}+\left(A_{s 1}-\gamma A_{s 2}\right) \dot{y}_{s 1}-\gamma Q_{s 2}-Q_{s l}(1-\gamma)}{\frac{V_{s 1}\left(x_{s}\right)}{\beta_{o}}+\frac{A_{s 1}^{2}}{K_{s T}}-\gamma^{2} \Delta} \ldots
$$

\subsubsection{Simulation Methodology}

Data of the implemented plant actuators for the Steckel mill under consideration are not available. Information of the physical constants and dimensions of such actuators was extracted from the literature. ${ }^{3,20,21)}$ The Steckel mill has servovalves with a natural frequency, $f_{s}$, of $51 \mathrm{~Hz}$ which corresponds to the operating frequency range of $60-100$ $\mathrm{Hz}$, listed by Guo. ${ }^{3)}$

A suitable value of $K_{v s}$ was not found in the literature and was calculated by observing that, $\sqrt{\left(2 / \rho_{o}\right) \Delta P_{s i}(t)}$ in Eq. (27) has units $\left(\left[\mathrm{m} \cdot \mathrm{s}^{-1}\right]\right)$, and $K_{v} x_{v_{s}}$ has the units of area. The rated flow of the valve has to pass through the servovalve into the cylinder and this area is taken as the spool drive area yielding, $K_{v s}=A_{\text {spool drive }} / x_{v_{\text {smax }}}=1.35 \mathrm{~cm}^{2} / 3 \mathrm{~mm}=$ $0.045 \mathrm{~m}$

It is assumed that the response of the second order transfer function servovalve model is critically damped. Once the spool movement is larger than $100 \%$ the flow, associated with a certain pressure drop, can not exceed the maximum flow, and a stroke greater than $100 \%$ will not reflect this limitation. This phenomena was incorporated as a hard constraint on the stroke length of $\pm 3 \mathrm{~mm}$ in the mill simulator. In Fig. 9 the cascaded controlled servovalve-spool strokes with the $|3 \mathrm{~mm}|$ hard limit are shown. Thus if the

\footnotetext{
*16 i) The servovalve; ii) The hydraulic transmission line; iii) The hydraulic cylinder; iv) The mill (dynamics, natural frequency, modulus and damping effect
} forming a compliant structure, v) The return line; vi) The position or pressure sensor. 
Table 2. Thrust force capability.

\begin{tabular}{|c|c|c|}
\hline$P_{s 1}(\mathrm{MPa})$ & $P_{s 2}(\mathrm{MPa})$ & $F_{H A_{s}}(\mathrm{MN})$ \\
\hline \hline 5 & 5 & 2.9 \\
\hline 5 & 35 & -1.9 \\
\hline 35 & 5 & 25.1 \\
\hline 35 & 35 & 20.3 \\
\hline
\end{tabular}

gain of the cascaded PI stroke position controllers of the hydraulic systems are adjusted such that the servovalve spools' limits are readily attained for larger required strokes, the system response will be slower than anticipated due to the flow saturation. A critically damped second order response was modelled, but in Fig. 9 an underdamped simulated response was obtained emphasizing the nonlinearity of the simulator.

The thrust force capability of the modelled actuators is given in Table 2, and a physical constraint of $40 \mathrm{MN}$ ( $20 \mathrm{MN}$ on each side of the mill) is applied. The modelled actuators are therefore capable of handling these rolling forces.

\subsection{Model Incorporation}

The TM and a combination of the HA-models and the SM simulate the dynamic behaviour of the simulator. The combination of the hydraulic actuator models and the stand model is expressed as a nonlinear state-space model. This nonlinear state-space model is solved using a fourth order fixed time step Runge Kutta numerical integration method. ${ }^{19)}$ The nonlinear state-space model has 18 states in total and the tension model has 2 states expressed in integral state-space format. In Fig. 10 the flow chart of the mill simulator is shown. This high level flow chart shows how the various models are used in the mill simulator.

The assignment of states $\mathbf{x}$ for the nonlinear state-space model is as follows:

$$
\begin{array}{cccccc}
\left(x_{1}\right. & \cdots & x_{8} & x_{9} & \cdots & \left.x_{18}\right)^{T}= \\
\left(q_{1_{1}}\right. & q_{1_{2}} & q_{2_{1}} & q_{2_{2}} & \dot{q}_{1_{1}} & \dot{q}_{1_{2}} \\
\dot{q}_{2_{1}} & \dot{q}_{2_{2}} & x_{v_{L}} & \dot{x}_{v_{L}} & P_{L 1} & x_{v_{R}} \\
\dot{x}_{v_{R}} & P_{R 1} & P_{L 2} & P_{R 2} & x_{L} & \left.x_{R}\right)^{T} .
\end{array}
$$

The first eight states shown in Eq. (32) are used with the linear model shown in Eq. (20). The states of the nonlinear state-space model (see Table 3), of the mill stand and hydraulic actuators, was calculated in order to have a bumpless start up of the simulator. The simulator is started on the acceleration speed ramp of the mill drive synchronous motor, and thus the simulation only starts after a part of the pass has been completed.

The states at steady state were calculated by taking the calculated rolling force at $t_{\text {Main }}=0$. This calculated rolling force was divided by two reflecting both sides of the mill to yield the shearing forces at the edges of the top roll pack. In the steady state these shearing forces must equal the thrust forces of the hydraulic actuators. From these thrust forces the pressures in the cylinder chambers can be calculated, by setting the leakage flow between the chamber 1 and 2 to zero. This implies that the pressures of the main and secondary chambers are equal.

The shearing forces at the edges of the bottom roll pack compresses the load cells and are taken as the vertical dis-

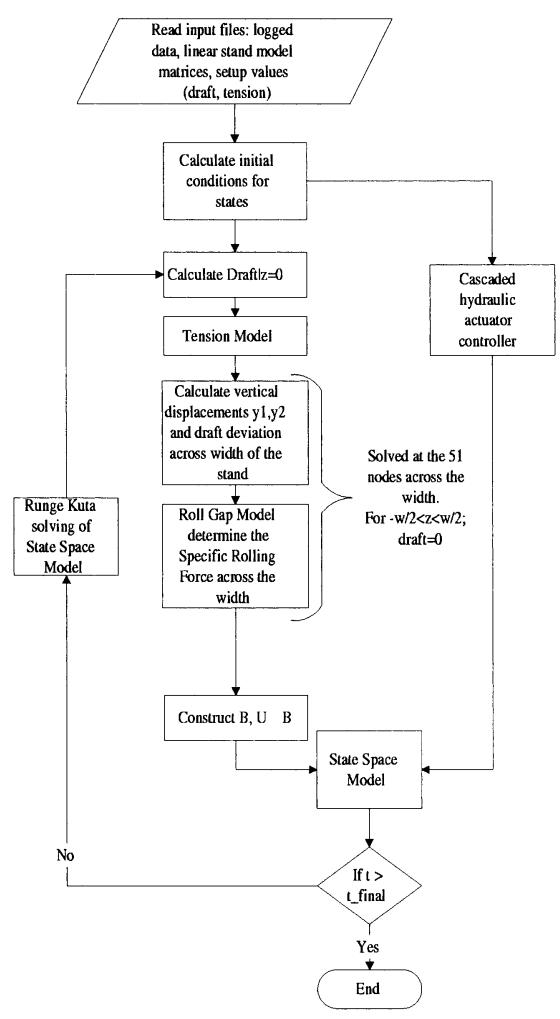

Fig. 10. Mill simulator flowchart.

Table 3. Calculated states for bumpless simulation start up. The units of the states are not shown.

\begin{tabular}{|c|c|}
\hline State & Value \\
\hline \hline$x_{1}, x_{2}, x_{5-10}, x_{12}, x_{13}$ & 0 \\
\hline$x_{3}$ & $1.84 \times 10^{-6}$ \\
\hline$x_{4}$ & $-3.12 \times 10^{-5}$ \\
\hline$x_{7}$ & $-3.12 \times 10^{-5}$ \\
\hline$x_{11}, x_{14-16}$ & $2.15 \times 10^{7}$ \\
\hline$x_{17}$ & $3.68 \times 10^{-3}$ \\
\hline$x_{18}$ & $3.65 \times 10^{-3}$ \\
\hline
\end{tabular}

placement of the bottom roll pack. The vertical displacement is used to calculate the initial states associated with the vertical displacements of this roll pack. It is assumed that the top roll pack does not have an initial vertical displacement, and the states associated with this roll pack are all taken as zero.

A static BISRA-Davy gaugemeter compensation compensates for this mill stretch, and the steady state values of the dynamic hydraulic strokes are increased with the relevant compensation quantities, on either side of the mill.

\section{SID and Simulation Results}

SID is used on practical plants to obtain models by analysing response data obtained after the application of certain signals to the plant. ${ }^{22)}$ An algebraic linearization of the derived models, might prove insufficient to capture the implicit process behaviour and SID was chosen as the means of identifying linear models from the simulator.

The controlled variables ${ }^{* 17}$ of the MIMO system, and the manipulated variables ${ }^{* 18}$ are indicated in Fig. 5. The hy-

$*_{17} \mathbf{y}(\mathbf{s}) \triangleq\left[\begin{array}{lll}\delta h_{2}(s) & \delta T_{1}(s) & \delta T_{2}(s)\end{array}\right]^{\mathrm{T}}$

$* 18 \mathbf{u}(s) \triangleq\left[\begin{array}{lll}\delta x_{s p_{L \& R}}(s) & \delta v_{b c}(s) & \delta v_{f c}(s)\end{array}\right]^{\mathrm{T}}$. 


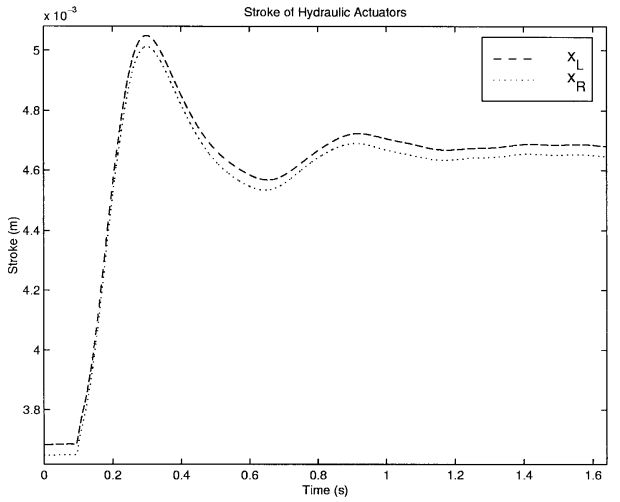

Fig. 11. Hydraulic strokes, after the application of a step $\left.\delta x_{s p_{L \& R}}\right|_{t \geq 92.6 \mathrm{~ms}}=1 \mathrm{~mm}$.

draulic actuators function as a combined unit.

The linearized model can be expressed in Laplace transform domain ${ }^{16)}$ as ${ }^{* 19}$,

$$
\mathbf{y}(s)=\mathbf{G}(s) \mathbf{u}(s)+\mathbf{G}_{d}(s) \mathbf{d}(s) .
$$

where $d(s) \triangleq\left[\begin{array}{lll}\delta h_{1}(s) & \delta T_{1}(s) \delta T_{2}(s)\end{array}\right]^{T}$ are the measured disturbances; $g_{i j}(s) \in \mathbf{G}(s)$ is the plant transfer function matrix with elements between the $i$ th output and $j$ th input; $g_{d i j}(s) \in \mathbf{G}_{d}(s)$ is the disturbance transfer function matrix with elements between the $i$ th output and $j$ th disturbance.

The linear plant model is obtained by applying step tests to one of the three manipulated inputs whilst the other two inputs are kept at their steady state operating points. The main mill drive speed is forced constant in the simulator to eliminate any possible dynamic effects on the controlled outputs due to $\delta v_{\text {roll }}$.

The data of the applied step tests were analyzed with the aid of the Matlab System Identification Toolbox. ARX (Auto Regression with eXternal input) linear models were fitted. $^{23)}$

The magnitude of the hydraulic stroke step was chosen as $1 \mathrm{~mm}$. From Fig. 5 in Ref. 8) the response of a supervisory GPC-controller used to regulate the rolling process the manipulated stroke was rarely larger than $1 \mathrm{~mm}$ in the presence of unmeasured disturbances. ${ }^{8}$ )

In Fig. 11 the second order time response of the hydraulic strokes of both actuators are shown. For this 2 nd order response $(\omega \approx 26 \mathrm{rad} / \mathrm{s}, \zeta \approx 0.3)$ a bandwidth of $6 \mathrm{~Hz}$ was calculated which is less than a value of $15 \mathrm{~Hz}^{7)}$ specified as a typical value for a hydraulic system.

In Fig. 12 a three dimensional view of the top and bottom roll pack's small signal movements are shown after the application of a hydraulic stroke setpoint change at $t=92.6 \mathrm{~ms}$. The bottom roll pack stays almost static (ignoring the high frequency small amplitude vibrations), while the top roll pack moves up and down in a controlled fashion. The high frequency oscillations can be attributed to a variety of effects. For instance the simulator was not started from exactly steady state and the allowed time before the application of the forcing step was not sufficient for the system to settle to the steady state. Another contributing effect is that the higher frequency tension process might change the roll separating force via the roll gap model in such a manner that high frequency oscillations are exhibited. It needs to be stressed that damping was only modelled be-

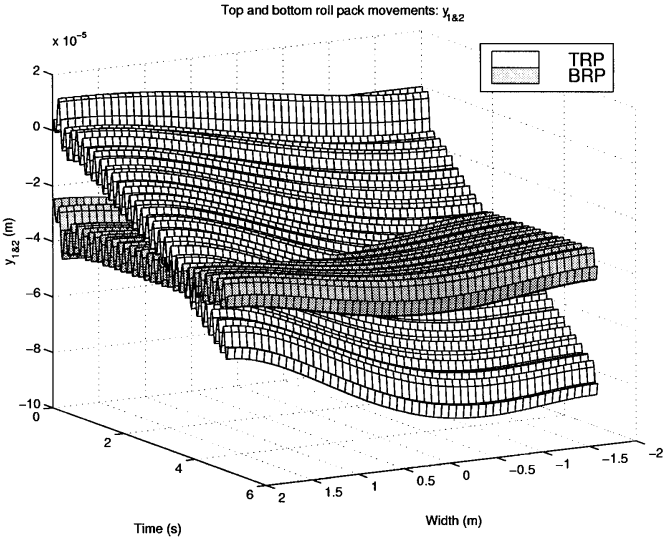

Fig. 12. Three dimensional view of the small signal movement of the roll packs as functions of space and time, after the application of a hydraulic stroke step input $\left.\delta x_{s p_{L \& R}}\right|_{t \geq 92.6 \mathrm{~ms}}=1 \mathrm{~mm}$.

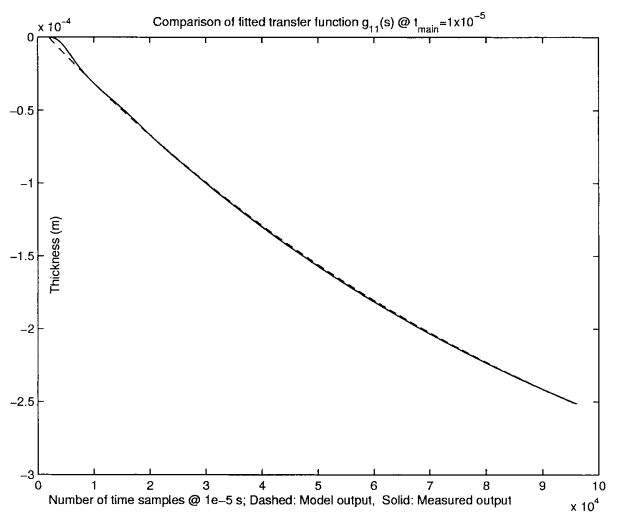

Fig. 13. Fitted model compared to the simulation data for $g_{11}(s)$.

tween the strip and the roll-packs, although the hydraulic jacks might contribute to the overall damping of the system. Thus the high frequency vibrations shown in Fig. 12 might be damped out when the models of the hydraulic jacks are included in the simulator.

The centerline exit gauge-hydraulic stroke transfer function will be the most important when a model based controller is implemented to predict the behaviour of the exit gauge due to a change in the hydraulic stroke. The transfer function is calculated as,

$$
g_{11}(s)=\frac{-8.0865 \times 10^{-2}}{s+1.9 \times 10^{-1}} e^{-4.15 \times 10^{-3} s} .
$$

In Fig. 13 the fitted transfer function model is compared against the simulation data obtained from the nonlinear plant simulator. The accuracy of the identified transfer function models were evaluated using the autocorrelation function of the error between the model output and the simulation data, and the cross correlation between this error and the manipulated variable. ${ }^{9,23)}$

The speed steps were calculated in order to have the maximum effect on the centerline gauge. This entails varying the tensions between 0 and $200 \mathrm{MPa}$. The step size of the coiler speed and the time duration were chosen to cover the specified range, and $\Delta v_{c}= \pm 0.2 \mathrm{~m} / \mathrm{s}^{* 20}, \Delta t_{\text {step }}=0.06 \mathrm{~s}$ were calculated using the relation, ${ }^{9}$ )

*19 $g_{d 11}(s), g_{d 21}(s), g_{d 23}(s), g_{d 31}(s), g_{d 32}(s)$ falls outside the scope of this work, and $g_{d 23}(s), g_{d 32}(s) \approx 0$, because the tensions are connected via a static roll gap

model. 


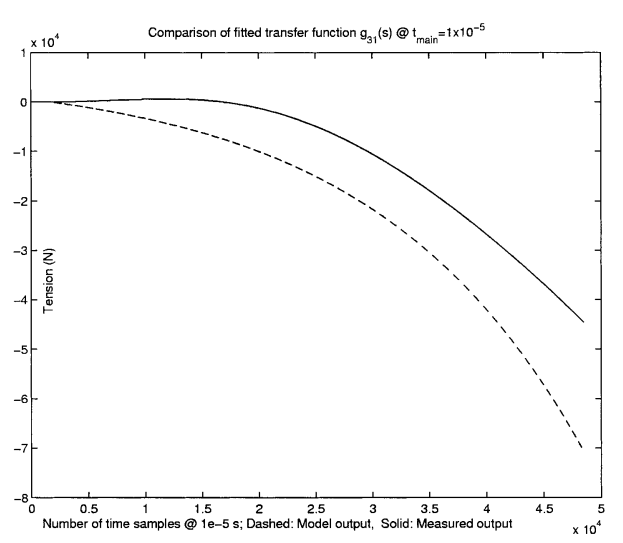

Fig. 14. Fitted model compared to the simulation data for $g_{31}(s)$.
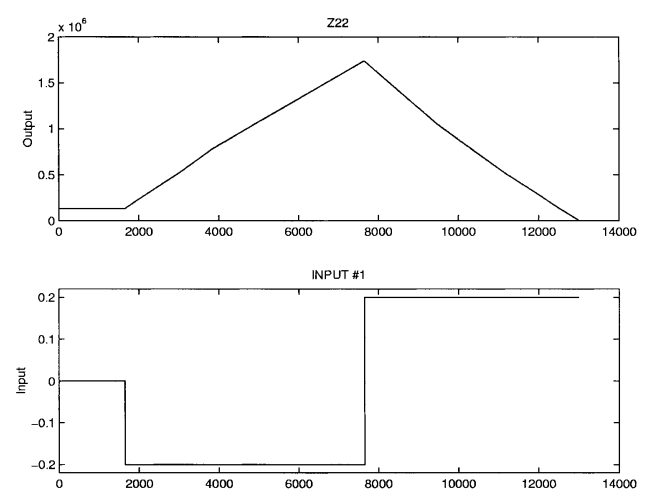

Fig. 15. The input/output relationship for the derivation of $g_{22}(s)$.

$$
E_{s s} h_{i} w L_{r g \leftrightarrow c f}^{-1} \Delta v_{c} \Delta t_{\text {step }}=2 \times 10^{8} h_{i} w-T_{i_{\text {setup }}}
$$

In Fig. 14 the identified transfer function model for $g_{31}(s)$ is compared to the nonlinear plant simulation data. The stroke setpoint to tension transfer functions are,

$$
\begin{aligned}
& g_{21}(s)=\frac{1.1 \times 10^{5} s-2.1 \times 10^{10}}{s^{2}+2.1 \times 10^{2} s-2.1 \times 10^{3}} e^{-0.15 \times 10^{-3} s}, \ldots \\
& g_{31}(s)=\frac{3.5 \times 10^{5} s-7.0 \times 10^{10}}{s^{2}+2.2 \times 10^{3} s-1.2 \times 10^{4}} e^{-0.33 \times 10^{-3} s} \ldots
\end{aligned}
$$

The speed to tension transfer functions were calculated also see Fig. 15,

$$
g_{22}(s)=-\frac{308 \times 10^{6}}{s}, \quad g_{33}(s)=\frac{225 \times 10^{6}}{s} \ldots
$$

In Ref. 9) it is seen that for small speed steps the influence on the thickness output is small. The speed inputs do play a role as soon as the tension start to near the yield stress of the material. As an illustration of the effect that tension has on gauge, $T_{1}$ was stepped and the corresponding transfer function is shown in Eq. (41). In Ref. 9) it was found that for short and small tension steps the influence on the exit gauge is small. On the other hand if a large tension step is applied it was seen that the influence on the exit gauge can become more significant. The rest of the transfer functions are,

$$
g_{12}(s)=\frac{8.0 \times 10^{-5} s^{2}+2.0 s-5.9 \times 10^{4}}{s^{3}+6.9 \times 10^{4} s^{2}+8.5 \times 10^{8} s-3.8 \times 10^{10}} e^{-5 \times 10^{-3} s},
$$

$$
\begin{aligned}
& g_{32}(s)=\frac{4.3 \times 10^{5} s^{2}-6.8 \times 10^{10} s-1.2 \times 10^{14}}{s^{3}+1.6 \times 10^{5} s^{2}-3.2 \times 10^{6} s+2.1 \times 10^{8}} e^{-3.2 \times 10^{-3} s}, \\
& g_{d_{12}}(s)=\frac{-5.28 \times 10^{-11}}{s+1.96 \times 10^{-1}}
\end{aligned}
$$

\section{Control Problem}

The thickness controller must be able to reject disturbances such as non-uniform input thickness, hardness variations, input and output tension variations and friction variations whilst maintaining an uniform output gauge. ${ }^{7,8)}$ Fast roll gap movements introduced to correct thickness disturbances generate transient tension changes. These result in uncontrolled thickness variations if no tension control is done. Thickness variations not only have an adverse effect on quality and profit but may also lead to mill instability during the rolling of later passes and subsequent cold rolling.

The most commonly employed control scheme for thickness control and compensation for mill stretch is a combination of the BISRA-Davy gaugemeter*21, and a closed loop thickness controller. A transport delay in the feedback path, associated with the X-ray measurement of $h_{2}$, can make the feedback control system unstable and a Smith Predictor is used to compensate for this delay. ${ }^{7)}$

The proposed method for further investigation is MPC, and its IMC structure makes it possible to predict how the plant will react to a certain input vector sequence in a receding time horizon framework. ${ }^{22)}$ At each time step an optimal control input sequence is computed using a quadratic programming technique, although only the first elements of the multivariable sequence are applied. In order to apply MPC, bounds for the manipulated, as well as their derivatives, and controlled variables need to be specified.

From measured plant data a typical thickness controller is capable of regulating the centerline gauge with a standard deviation of $\sigma_{y_{1}}=0.02 \mathrm{~mm}$ for the chosen operating point. From this data nothing can be concluded regarding tension constraints.

The output constraint imposed on the output gauge is, $\left|y_{1}\right| \leq 60 \mu \mathrm{m}$. The tension output limits are specified as,

$$
0 \leq y_{i+1}<k(\bar{\varepsilon}, \overline{\dot{\varepsilon}}, \bar{\theta}) w h_{i}, \quad i \in[1,2] .
$$

The tension bounds are conservative which might yield an ill posed optimization problem, and these bounds need some revisiting.

The bound on the combined dynamic hydraulic stroke is, $\left|u_{1}\right| \leq 1 \mathrm{~mm}$, which reflects the domain of linearization. Higher order rate constraints suggested in Ref. 7) for a $15 \mathrm{~Hz}$ hydraulic system are $\left|\dot{u}_{1}\right| \leq 4 \mathrm{~m} \cdot \mathrm{s}^{-1}$, and $\left|\ddot{u}_{1}\right| \leq$ $500 \mathrm{~m} \cdot \mathrm{s}^{-2}$. Although the hydraulic systems were found to have a bandwidth of $6 \mathrm{~Hz}$, the latter constraints are still proposed.

The limits on the coiler speed inputs reflect the domain of linearization, and are $\left|u_{2 \& 3}\right| \leq 0.2 \mathrm{~m} \cdot \mathrm{s}^{-1}$. Each synchronous machine has a limited amount of torque available to accelerate its load. The load can not be accelerated immediately $\left(\dot{u}_{i}(t) \neq \infty, \forall i \in[2,3]\right)$ due to the large inertia associated with the strip and coiler bobbin combination as well as the rotor of the machine. Before applying MPC, further in-

*21 This compensator employs positive force feedback to regulate $h_{2}{ }^{7)}$ 
vestigations are proposed to identify the relevant mechanical constants of the machine swing equations and the subsequent constraints.

Note that this constrained optimization problem at each time step can impede the performance of the controller, because the amount of time required to solve the optimization problem might be long compared to the process time constants. MPC is usually applied in the chemical process industry where the times constants of the processes are longer than the time constants associated with the rolling process. If it is found that the constrained problem can not be solved in the allocated time frame, it might be beneficial to relax the constraints. GPC (Generalized Predictive Control)*22 was applied to a rolling mill in Ref. 8), ultimately demonstrating the feasibility of predictive control methods in the rolling mill environment.

\section{Conclusion}

In this paper a hot rolling Steckel mill was modelled in order to yield a nonlinear plant simulator. Process behaviour was modelled in order to reflect centerline gauge behaviour, thickness crown behaviour and tensions in the strip on either side of the roll gap. The mill simulator was used to identify a LTI MIMO transfer function model, that can be used for control system design using methods such as MPC.

South Africa has at least three hot rolling Steckel mills making this work applicable to the South African rolling industry. The creation of a simulator of a Steckel mill, modelled according to an existing practical mill that agrees acceptably with the literature and practical data, is seen as a contribution given the absence of extensive literature on Steckel mills.

\section{Nomenclature}

$\bar{\varepsilon}([\mathrm{m} / \mathrm{m}]):$ mean strain;

$\overline{\dot{\varepsilon}}=\frac{\ln \left(h_{1} / h_{2}\right) v_{\text {roll }}}{\sqrt{R^{\prime} \delta}}\left(\left[\mathrm{s}^{-1}\right]\right):$ mean strain rate;

$\theta\left(\left[{ }^{\circ} \mathrm{C}\right]\right): \quad$ strip temperature;

$R([\mathrm{~m}])$ : roll radius;

$R^{\prime}([\mathrm{m}])$ : deformed roll radius;

$\phi([\mathrm{rad}])$ : angle of the arc of contact, measured from the exit of the roll gap;

$\phi_{m} \approx \sqrt{\delta R^{\prime-1}}:$ bite angle;

$\sigma_{i}([\mathrm{~Pa}])$ : flow stress at the entrance/exit of the roll gap;

$Q_{Z H}$ : activation energy for onset of plastic deformation;

$m$ : strain rate sensitivity exponent;

$n$ : work hardening exponent;

$R_{\text {ideal }}:$ ideal gas constant;

$A_{Z H}$ : material constant used in the ZenerHoloman model;

$v$ : Poisson's ratio for steel;

$E$ : Young's modulus of the work rolls;

$A_{\varrho}=A_{\chi B R}+A_{\chi W R}:$ area of roll pack $(\chi \in[T, B])$; $I_{\varrho}=I_{\chi W R}:$ second moment of area of roll pack, ${ }^{2)}$ $K_{s T} \approx 3 \times 10^{9} \mathrm{~N} / \mathrm{m}: \quad$ linear mill spring approximation;

$$
\beta=\frac{\left.k(\varepsilon, \dot{\varepsilon}, \theta)\right|_{x_{r g}=0}}{\omega_{\text {main }}}\left[\frac{\mathrm{N}}{\mathrm{m}^{2} \cdot \mathrm{s}^{-1}}\right]: \text { distributed damping }
$$

coefficient between the rolls and the strip;

$\left.k(\varepsilon, \dot{\varepsilon}, \theta)\right|_{r_{r q}=0} \approx 280 \mathrm{MPa}$ : yield stress at the delivery side of the roll gap;

$\omega_{\text {main }}$ : main mill drive angular velocity;

$x_{v}$ : servovalve opening (spool movement);

$x_{v_{\text {s }}}=3 \times 10^{-3}:$ maximum spool movement;

$x_{s}$ : hydraulic stroke setpoint;

$\omega_{v_{s}}=2 \pi \times 51 \mathrm{rad} \cdot \mathrm{s}^{-1}$ : servovalve natural frequency;

$\zeta_{v}=1: \quad$ servovalve damping ratio;

$I_{c}$ : controlled input current;

$I_{o_{s}}= \pm 10 \mathrm{~mA}^{c_{s}}:$ rated servovalve current;

$Q_{s 1}, Q_{s 2}: \quad$ cylinder flow into/out the piston/rod side;

$P_{s 1}, P_{s 2}: \quad$ cylinder pressure on the piston/rod side; $Q_{s 1}=K_{\text {leak }_{s}}\left(P_{s 1}-P_{s 2}\right):$ leakage flow;

$x_{s}$ : hydraulic stroke movement;

$V_{0 s t}:$ initial oil volumes in the chambers;

$A_{s 1}, A_{s 2}$ : area on the piston/rod side;

$K_{\text {leak }}$ : leakage flow coefficient;

$\rho_{o}$ : density of the hydraulic oil;

$\beta_{o}$ : oil bulk modulus.

\section{REFERENCES}

1) A. Lederer: Metall. Plant Technol. Int., 16 (1993), 56.

2) L. M. Pederson and B. Wittenmark: IEEE Trans. Control System Technology, 6 (1998), 304.

3) R.-M. Guo: Iron Steel Eng., (1991), 52.

4) G. Hearns and M. J. Grimble: Preprints of the Proc. of the 9th IFAC MMM Conf., IFAC, Elsevier Science, Oxford, (1998), 215.

5) R.-M. Guo: Iron Steel Eng., (1990), 32.

6) C. Devadas, I. V. Samarasekera and E. B. Hawbolt: Metall. Trans. A, 22 (1991), 307

7) M. J. Grimble, M. R. Katebi, G. Hearns and M. A. Johnson: International Training Course in Hot Rolling Mill Control and Technology. Industrial Control Centre (University of Strathclyde UK), (1998).

8) M. J. Grimble, M. R. Katebi: IFAC Automation in the Steel Industry, Kyongju, Korea, IFAC, Elsevier Science, Oxford, (1997).

9) E. Scholtz: Modelling for Control of a Steckel Hot Rolling Mill Process. Master's thesis, Department of Electrical and Electronic Engineering, University of Pretoria, (1999).

10) F. R. Camisani-Calzolari, Z. M. Smit, I. K. Craig and R. Torr: IEEE Int. Symp. on Industrial Electronics, Pretoria, South Africa, IEEE, Piscataway, NJ, (1998).

11) B. T. Boulter: IEEE Trans. Industry Applications, 35 (1999), 126

12) E. Orowan: Proc. of the Institution of Mechanical Engineers, 150 (1943), 140.

13) G. E. Dieter: Mechanical Metallurgy, McGraw-Hill, New York, (1986).

14) W. L. Roberts: Hot Rolling of Steel, Marcell Dekker, New York, (1983).

15) E. Scholtz, I. K. Craig and P. C. Pistorius: Proc. of IEEE CCA/CACSD 2000 Conf. IEEE, Piscataway, (2000).

16) G. Stephanopoulos: Chemical Process Control: An Introduction to the Theory and Practice, Prentica Hall International Editions, (1984).

17) W. Dobrucki and A. Bar: J. Mater. Process. Technol., 61 (1996), 328.

18) L. Meirovitch: Analytical Methods in Vibrations, Macmillan, New York, (1967).

19) J. H. Matthews: Numerical Methods for Mathematics, Science and Engineering, Prentice Hall International Editions, 2 ed., (1992).

20) V. B. Ginzburg: AISE Year Book, (1984), 75

21) P. Bhowal and S. K. Mukherjee: ISIJ Int., 36 (1996), 553.

22) M. Morari and J. H. Lee: Computers and Chemical Engineering, 23 (1999), 667.

23) L. Ljung: System Identification Toolbox, for use with matlab, (1997).

*22 A similar method to MPC. 